Running head: DYNAMIC SUPERIORITY EFFECT

When are moving images remembered better? Study-Test Congruence and the Dynamic Superiority Effect

Luciano G. Buratto

William J. Matthews

Koen Lamberts

University of Warwick, UK

Word count: 3951 words

E-mail: 1.g.buratto@,warwick.ac.uk

Phone: +44-(0)2476-522-774

Address:

Department of Psychology

University of Warwick

COVENTRY

CV4 7AL

United Kingdom 


\begin{abstract}
It has previously been shown that moving images are remembered better than static ones. In two experiments, we investigated the basis for this dynamic superiority effect. Participants studied scenes presented as a single static image, a sequence of still images, or a moving video clip, and 3 days later completed a recognition test in which familiar and novel scenes were presented in all three formats. We found a marked congruency effect: For a given study format, accuracy was highest when test items were shown in the same format. Neither the dynamic superiority effect nor the studytest congruency effect were affected by encoding (Experiment 1) or retrieval (Experiment 2) manipulations, suggesting that these effects are relatively impervious to strategic control. The results demonstrate that the spatio-temporal properties of complex, realistic scenes are preserved in long-term memory.
\end{abstract}

Keywords: recognition, long-term memory, pictures, motion, perceptual specificity 


\section{When are moving images remembered better? Study-Test Congruence and the Dynamic Superiority Effect}

Research on recognition memory for complex visual stimuli has mainly focused on how people remember static images (e.g., Lamberts, Brockdorff, \& Heit, 2002). However, our environment is intrinsically dynamic. Therefore, to understand the processes that underlie visual memory in everyday life, it is important to establish the properties of memory for dynamic stimuli. Dynamic information has been shown to improve the identification and categorisation of people's actions (e.g., Mather \& Murdoch, 1994) and to facilitate the learning and recognition of faces (e.g., Lander \& Bruce, 2003; O'Toole, Roark, \& Abdi, 2002). Recently, Matthews, Benjamin and Osborne (2007) found that dynamic information may also aid long-term recognition memory. In two experiments, moving scenes (film clips presented at 25 frames per second) were better recognised than multi-static scenes (clips at 2 frames per second) and static scenes (single frames chosen from a clip). In addition, the memory advantage for dynamic scenes persisted for retention intervals of up to a month. In this article, we aim to further explore this dynamic superiority effect.

In all conditions in Matthews et al. (2007), the mode of presentation at study was identical to the presentation mode at test: moving scenes at study were probed with moving scenes at test and static scenes at study were probed with static scenes at test. Consequently, Matthews et al.'s (2007) design does not permit further analysis of the origin of the observed dynamic superiority effect. The effect could depend on an advantage at encoding (e.g., more information stored at study with moving scenes), an advantage at retrieval (e.g., more cues available at test with moving scenes) or a combination of factors at encoding and retrieval (e.g., encoding specificity; Tulving, 1983).

In an earlier study, Goldstein et al. (1982) had demonstrated that recognition was best if dynamic pictures were presented both at study and at test, compared to two other conditions (one in which static study pictures were probed with dynamic test cues and another in which dynamic study pictures were probed with static cues), suggesting that the dynamic superiority effect may depend on the match between study and test conditions. It is difficult, however, to draw firm conclusions from Goldstein et al.'s study, because encoding times for moving and static pictures were not matched (e.g., static pictures were shown for $5 \mathrm{~s}$ at study whereas dynamic pictures were shown as a continuous stream for $10 \mathrm{~min}$ ). As a result, it is not possible 
to determine whether the dynamic advantage stemmed from differences in available study information or in available study time. Still, the data from Goldstein et al. (1982) suggest that the match between study and test conditions may modulate the dynamic superiority effect, and therefore merits further investigation.

The beneficial effects of encoding-retrieval similarity have been extensively reported in the literature (e.g., Tulving, 1983, pp. 226-238). The general finding is that matching conditions yield better recognition than mismatching conditions (giving rise to a perceptual specificity or study-test congruency effect). For example, pictures are better recognised at test if they have the same size as the originally studied ones (Rajaram, 1996). Similarly, pictures encoded in a particular viewing mode (foveal or parafoveal) are better recognised if presented at test in the same viewing mode (Reingold, 2002). The matching advantage is preserved even after a 48-hour retention interval (Ray \& Reingold, 2003).

For any class of stimuli, congruency effects involving perceptual features or perceptual processing modes indicate that not only semantic information but also perceptual information is encoded in long-term memory. Indeed, evidence from neuro-imaging, eye-tracking and chronometric studies converges on the notion that recognition of a wide range of stimuli involves the reinstatement of processes originally engaged at study (e.g., Kent \& Lamberts, 2008).

In Experiment 1 of the current study, we investigated the role of encoding and retrieval factors (and their interaction) in the dynamic superiority effect. In anticipation of the results, we observed strong study-test congruency effects, in addition to an overall dynamic superiority effect. In Experiment 2, we investigated whether the dynamic congruency effect depends on explicit storage and recall of information about presentation mode.

\section{Experiment 1}

To investigate the role of encoding and retrieval factors (and their interaction) in the recognition-memory advantage for moving pictures relative to static pictures, presentation modes at study (moving, multi-static and static) were crossed with presentation modes at test (also moving, multi-static and static). If the encodingspecificity principle in recognition memory (Tulving, 1983) extends to the spatiotemporal domain, conditions with matching presentation modes at study and at test should produce better memory performance than conditions with different modes at study and test. 
We also assessed whether the dynamic advantage depends on the engagement of intentional encoding strategies. In the study phase, participants were either warned (intentional condition) or not (incidental condition) about the upcoming memory test. To discourage deep semantic processing, which could mask potential effects of intentionality, all participants undertook a "shallow" encoding task, in which they had to decide whether there was a woman in the scene. Gender identification has been shown to induce superficial processing in studies of face recognition (Bower \& Karlin, 1974).

\section{Method}

Participants. Seventy-two University of Warwick students (30 males; age: $M$ $=24.3, S D=6.8$ ) participated in the study. There were thirty-six participants in each encoding condition (incidental or intentional). The participants were paid $£ 12$ each.

Materials. Stimuli consisted of 540 video clips extracted from a collection of unfamiliar films and documentaries. Nine clips were constructed from each of 60 films. Care was taken to minimise the overlap of characters in clips extracted from the same film. The clips were silent and presented in black and white with no cuts and no camera movement. They all featured people, men and women, from a broad range of countries, ages and epochs, talking, moving and interacting with each other.

Each clip was presented for $3 \mathrm{~s}$. Three presentation modes were used: moving, multi-static, and static. Moving stimuli were constructed by playing 75 frames ( 25 per second) to a total of $3 \mathrm{~s}$. Multi-static stimuli were constructed by taking frames 1, 15, 30,45 and 60 from the clip and presenting each frame for $600 \mathrm{~ms}$ in chronological order and without gaps. The static stimuli consisted of a single frame shown for $3 \mathrm{~s}$ and selected at random from the 75 possible frames.

The video clips were divided into 18 sets of 30 clips. The sets were matched for average complexity and visual properties. Complexity was evaluated in terms of file size (png and jpg formats), compression ratio (bmp file size relative to jpg size) and edge density (the percentage of edge pixels) (Rosenholtz, Li, \& Nakano, 2007; Szekely et al., 2005). The sets of 30 clips were then randomly allocated to encoding conditions and presentation modes.

Design and Procedure. Participants first took part in a study phase in which they were presented with a sequence of 270 clips (90 moving, 90 multi-static and 90 static, all intermixed). In the incidental encoding condition, participants were simply 
told to look for a woman in the scene. After seeing the clip, they should enter a "yes" or "no" response by pressing one of two keys. In the intentional encoding condition, participants were also informed about the subsequent memory test.

Participants in both encoding conditions returned three days later for the test phase. The test consisted of 540 trials. Half of the test trials contained a new clip (i.e., a clip not seen in the study phase, in any presentation mode), whereas the other test trials contained an old clip (i.e., a clip presented in the study phase, possibly in a different presentation mode). New clips were split into equal numbers of moving, multi-static and static clips. Similarly, the 270 old clips consisted of 90 moving, 90 multi-static and 90 static clips. For each set of 90 old clips, 30 were re-presented in their original mode (e.g., moving), and 30 were presented in each of the two different modes (e.g., multi-static and static). As a result, the within-subjects manipulations in the experiment produced an orthogonal $3 \times 3$ design, fully crossing presentation mode at study (moving, multi-static, and static) with presentation mode at test.

For each test clip, participants had to decide on a 6-point scale (from "definitely old" to "definitely new") whether or not the clip had been presented in the study phase. Participants were told that presentation mode was irrelevant for their decision, so that if they recognised a static frame from a moving study clip (for instance), they should respond “old”. No feedback on responses was provided. Results and Discussion

Recognition accuracy was computed with $d_{a}$, which is a multi-point sensitivity measure that controls for shifts in response bias (Macmillan \& Creelman, 2005). The use of $d_{a}$ thus allows comparisons of performance across conditions that differ in bias. Sensitivity was estimated for each participant in each condition by fitting a maximumlikelihood unequal-variance Gaussian model to ROC (Receiver Operating Characteristic) curves. The ROC curves were generated on the basis of participants' response ratings (see Buratto \& Lamberts, 2008).

Sensitivity $\left(d_{a}\right)$. A $3 \times 3 \times 2$ mixed-design ANOVA was carried out on $d_{a}$ with study mode (moving, multi-static and static) and test mode (moving, multi-static and static) as within-participant factors and encoding condition (incidental, intentional) as a between-subject factor. Because encoding condition did not interact with the other factors and did not yield a main effect (all $F \mathrm{~s}<1.12, p s>.35$ ), $d_{a}$ results were collapsed across encoding conditions. The ANOVA revealed a main effect of study 
mode, $F(2,140)=79.33, M S E=0.05, p<.001, \eta_{p}^{2}=.53$. Post-hoc LSD tests showed that sensitivity was higher for moving stimuli $(M=1.09, S E=0.05)$ than for multistatic stimuli $(M=0.97, S E=0.05)$, which in turn yielded significantly higher sensitivity than static stimuli $(M=0.81, S E=0.04)$. The fact that performance in the multi-static condition was intermediate between performance in the moving and static conditions suggests that the amount of information available at encoding plays a role in memory for moving pictures. There was also a main effect of test mode, $F(2,140)=$ $12.06, M S E=0.09, p<.001, \eta_{p}^{2}=.15$. Sensitivity was lowest when static scenes were presented at test $(M=0.88, S E=0.05)$ compared to moving $(M=0.99, S E=0.05)$ and multi-static $(M=1.01, S E=0.05)$ scenes, which did not differ from each other $(p$ $=.44)$.

Importantly, the ANOVA also revealed a study-test congruency effect, as a strong interaction between study and test presentation modes, $F(4,280)=25.12, M S E$ $=0.06, p<.001, \eta_{p}^{2}=.26$. The mean discriminability values in Figure 1 indicate that, for each study mode, test performance was best if there was a match between study and test modes. ${ }^{1}$

The dynamic superiority effect - the sensitivity advantage for moving scenes relative to multi-static and static scenes in congruent study-test conditions - was significant, $F(2,140)=35.10, M S E=0.06, p<.001, \eta_{p}^{2}=.33$, and the effect was not modulated by intentionality $(F<1, p=.51)$. Sensitivity was higher in the moving/moving condition $(M=1.25, S E=0.06)$ than in the multi-static/multi-static condition $(M=1.08, S E=0.06)$, in which sensitivity was higher than in the static/static condition $(M=0.91, S E=0.06)$.

Experiment 1 thus yielded four main results. First, the dynamic superiority effect, previously reported by Matthews et al. (2007) and Goldstein et al. (1982), was replicated: moving pictures were better recognised than static pictures. Second, the main effect of study mode suggests that the amount of information present at encoding played a role. The presence of more frames at study affords the storage of more instances or viewpoints of the same scene, possibly enhancing recognition through redundancy, or via the enhanced extraction of 3D structure or meaning. The main effect of test mode, in which stimuli presented in the static mode were less effective as retrieval cues, may have a similar explanation. Third, and most 
importantly, the dynamic advantage was modulated by the congruency between study and test conditions. For any study mode, discriminability was highest if the presentation mode at test matched the presentation mode at study. The study-test congruence effect provides strong evidence that the dynamic status of studied clips is retained in the memory representation. Priming studies with faces had already suggested that dynamic information is stored automatically (Lander \& Bruce, 2004), and our data extend these findings to a broader class of stimuli. Finally, neither the dynamic superiority effect nor the congruency effect depended on the intention to memorise at encoding, which suggests that both effects emerge from the representations that are constructed on the basis of spontaneous processing of complex visual stimuli.

\section{Experiment 2}

The congruence effect from Experiment 1 demonstrates that some form of dynamic status information is retained in memory, but it is not clear whether participants have conscious access to this information. To address this question, we

manipulated retrieval instructions. Participants were told either to respond "old" based solely on the content of the clip, regardless of presentation mode (inclusion condition) or to respond "old" based on both the content of the clip and presentation mode, thus saying "new" to a clip that had been presented in a different mode at study (exclusion condition).

According to dual-process models of recognition memory (Yonelinas, 2002), decisions at test can be based on the retrieval of context-dependent details about the studied item (recollection) or on a context-free feeling that the item has been seen before (familiarity). In the inclusion condition, recognition judgments could be based on familiarity or on recollection. In the exclusion condition, however, participants would have to rely on recollection in order to correctly accept targets (old clips with same mode at test) and to reject pseudo-targets (old clips with changed mode at test). Thus, the instructions in the exclusion condition should induce both recall-to-accept and recall-to-reject strategies at test (e.g., Rotello, Macmillan, \& Van Tassel, 2000). If the participants can consciously recollect mode information, they should be able to selectively accept targets and reject pseudo-targets based on the match between study and test presentation modes.

\section{Method}


Participants. Seventy-two students from the University of Warwick (32 males, mean age 23 years) took part in the study and were paid $£ 12$ each. There were 36 participants in each test condition (inclusion or exclusion).

Materials, Design and Procedure. The materials and study procedure were identical to that in the intentional coding condition from Experiment 1, except that participants were asked to decide for each clip on a 6-point scale whether they thought they would remember it in three days' time. In the inclusion condition, test instructions were identical to those in Experiment 1. In the exclusion condition, participants were told to respond "old" only when both the scene and the mode at test (moving, multi-static, static) were the same as in the clip seen at study. If, for example, a static frame was presented at test which was extracted from a clip presented in the moving mode at study, participants should respond "new".

\section{Results and Discussion}

Sensitivity $\left(d_{a}\right)$. In the inclusion condition, the sensitivity measures $d_{a}$ were computed in exactly the same way as in Experiment 1. In the exclusion condition, sensitivity was estimated in the same way for stimuli that were targets (old scenes tested in the same mode) and stimuli that were pseudo-targets (old scenes tested in a different mode, to which participants should respond "new"): $d_{a}$ was the standardised difference, corrected for unequal variance, between targets (or pseudo-targets) and unrelated lures (new scenes shown in the same mode as the corresponding target or pseudo-target). Therefore, for the correct interpretation of the results in the exclusion condition, it is important to bear in mind that the sensitivity measure for pseudotargets has a different meaning from the measure for targets. For pseudo-targets, high sensitivity would indicate that participants were able to discriminate between seen and unseen scenes, but were unable to use presentation mode as instructed. To avoid interpretation difficulties, the results from the inclusion and exclusion conditions were initially analysed separately.

For the inclusion condition, a $3 \times 3$ repeated-measures ANOVA was carried out on $d_{a}$ with study mode (moving, multi-static and static) and test mode (moving, multi-static and static) as the independent variables. The mean values for the different combinations of study and test mode are shown in Figure 2. The ANOVA revealed a main effect of study mode, $F(2,70)=35.85, M S E=0.09, p<.001, \eta_{p}^{2}=.50$ : sensitivity was higher for moving $(M=1.44, S E=0.09)$ than for multi-static clips $(M$ 
$=1.29, S E=0.08)$, which in turn exceeded sensitivity for static clips $(M=1.13, S E=$ 0.74; post-hoc Fisher's LSD test). In contrast with the results from Experiment 1, there was no main effect of test mode (moving: $M=1.30, S E=0.08$; multi-static: $M=$ 1.30, $S E=0.07$; static: $M=1.26, S E=0.09 ; F<1, p=.44)$. There was, however, an interaction between study and test mode, $F(4,140)=15.45, M S E=0.09, p<.001$, $\eta_{p}^{2}=.31$. For a given study mode, sensitivity was highest when the test presentation mode matched the study presentation mode, showing a congruency effect similar to that found in Experiment 1. The dynamic superiority effect was significant in the inclusion condition, $F(2,70)=10.84, M S E=0.10, p<.001, \eta_{p}^{2}=.24$ : sensitivity $d_{a}$ was higher in the moving/moving condition $(M=1.64, S E=0.12)$ than in the multistatic/multi-static condition $(M=1.41, S E=0.08)$ and static/static conditions $(M=$ 1.31, $S E=0.11)$. The difference between multi-static/multi-static and static/static conditions was not significant $(p=.15)$.

In the exclusion condition, a $3 \times 3$ repeated-measures ANOVA revealed a main effect of study mode, $F(2,70)=18.46, M S E=0.09, p<.001, \eta_{p}^{2}=.35$, showing that scenes studied in the moving mode $(M=0.99, S E=0.08)$ were better recognised than scenes studied in the multi-static $(M=0.86, S E=0.08)$ and static modes $(M=$ $0.75, S E=0.06)$. There was also a smaller effect of test mode, $F(2,70)=5.57, M S E=$ $0.22, p=.006, \eta_{p}^{2}=.14$, with recognition for scenes tested in the moving mode $(M=$ $0.97, S E=0.08$ ) being marginally better than for scenes tested in the multi-static mode $(M=0.86, S E=0.08 ; p=.07)$ and significantly better than for scenes tested in the static mode $(M=0.76, S E=0.08 ; p=.002)$. Performance did not differ between the multi-static and static test modes $(p=.15)$. The two-way ANOVA revealed a significant interaction between the study and test presentation modes, $F(4,140)=$ 20.53, MSE $=0.11, p<.001, \eta_{p}^{2}=.37$, again suggesting that discriminability was better when there was a match between study and test presentation modes. Figure 3 shows the means underlying this interaction. From the data in Figure 3, it is clear that the participants were often unable to reject pseudo-targets as instructed. Instead, the responses showed a congruency effect that was quite similar to that in the inclusion condition (and in Experiment 1), suggesting that the test instructions did not substantially alter the nature of the congruency effect. 
The results from an overall $3 \times 3 \times 2$ ANOVA on $d_{a}$ with study and test modes as within-participant factors and instruction (inclusion or exclusion) as a betweensubject factor are consistent with this suggestion. There was a main effect of instruction, $F(1,70)=16.59, M S E=1.75, p=.001, \eta_{p}^{2}=.19$, with higher sensitivity in the inclusion condition $(M=1.29, S E=0.07)$ than in the exclusion condition $(M=$ $0.86, S E=0.07)$, and there were the expected main effects of study and test mode and a study-test interaction $\left(F_{\mathrm{S}}>5.81, \mathrm{ps}<.01\right)$. More importantly, there was no threeway interaction between instruction, study mode and test mode $(F=1.25, p=.29)$.

\section{General Discussion}

In the two experiments, we replicated the dynamic superiority effect previously reported by Matthews et al. (2007), wherein moving pictures are better recognised than static pictures when study and test conditions are matched. Moreover, we showed that the memory advantage for moving pictures can be eliminated if the mode of presentation at test (e.g., static) mismatches the mode of presentation at study (e.g., moving). Finally, both the dynamic superiority effect and the study-test congruency effect were not affected by encoding and retrieval manipulations, suggesting that these effects are relatively immune to strategic control.

Why are moving pictures remembered better than static pictures? One possible reason is that moving pictures contain more information than static pictures. Indeed, sensitivity was higher when participants studied 75 frames (moving) compared to 6 frames (multi-static) and 1 frame (static), regardless of test mode. Similarly, moving pictures at test provide more cues to aid retrieval compared to the other presentation modes, producing better overall performance with dynamic cues at test, regardless of study mode. Another possible reason for the dynamic advantage is that moving pictures attract more attention and keep participants more engaged in the task than static pictures do. Previous research indicates that faces are better learned in non-rigid dynamic conditions (i.e., facial expressions without head movement) than in static conditions (Lander \& Davies, 2007) and that the dynamic advantage may be partly due to increased attention to non-rigidly moving faces (Lander \& Bruce, 2003).

Whereas information or attention differences can account for the dynamic superiority effect, they cannot explain the strong study-test congruency effects that we observed. The congruency effects suggest that the encoding of natural scenes involves the extraction not only of semantic information but also of dynamic 
information. Our results thus add to the growing body of evidence for perceptual specificity in recognition memory, which suggests that memory retrieval involves the simulation of processes originally engaged in the encoding of the stimulus (e.g., Kent \& Lamberts, 2008; Reingold, 2002).

Further research may shed light on the specific factors responsible for the dynamic superiority effect. It might be that the precise dynamic properties of moving pictures are important for the memory advantage, so that disrupting the natural flow of movement (for example, by playing clips in reverse) would lead to poorer performance. This manipulation would have the advantage of controlling for the amount of information (i.e., number of frames) in each condition. Alternatively, it may be possible to observe a dynamic advantage even with static stimuli that imply motion (e.g., a picture of someone throwing a frisbee) when compared to static stimuli that do not imply motion (e.g., a picture of the same person standing still). This manipulation would also allow for better control of the amount of information present in both "dynamic" and "static" conditions. 


\section{References}

Bower, G. H., \& Karlin, M. B. (1974). Depth of processing pictures of faces and recognition memory. Journal of Experimental Psychology, 103, 751-757.

Buratto, L., \& Lamberts, K. (2008). List strength effect without list-length effect in recognition memory. Quarterly Journal of Experimental Psychology, 61, 218226.

Goldstein, A. G., Chance, J. E., Hoisington, M., \& Buescher, K. (1982). Recognition memory for pictures: Dynamic vs. static stimuli. Bulletin of the Psychonomic Society, 20, 37-40.

Kent, C., \& Lamberts, K. (2008). The encoding-retrieval relationship: Retrieval as mental simulation. Trends in Cognitive Sciences, 12, 92-98.

Lamberts, K., Brockdorff, N., \& Heit, E. (2002). Perceptual processes in matching and recognition of complex pictures. Journal of Experimental Psychology: Human Perception and Performance, 28, 1176-1191.

Lander, K., \& Bruce, V. (2003). The role of motion in learning new faces. Visual Cognition, 10, 897-912.

Lander, K., \& Bruce, V. (2004). Repetition priming from moving faces. Memory \& Cognition, 32(4), 640-647.

Lander, K., \& Davies, R. (2007). Exploring the role of characteristic motion when learning new faces. Quarterly Journal of Experimental Psychology, 60, 519526.

Macmillan, N. A., \& Creelman, C. D. (2005). Detection theory: A user's guide (2nd ed.). Mahwah, NJ: Erlbaum.

Masson, M. E. J., \& Loftus, G. R. (2003). Using confidence intervals for graphically based data interpretation. Canadian Journal of Experimental Psychology, 57, 203-220.

Mather, G., \& Murdoch, L. (1994). Gender discrimination in biological motion displays based on dynamic cues. Proceedings of the Royal Society of London, 258, 273-279.

Matthews, W. J., Benjamin, C., \& Osborne, C. (2007). Memory for moving and static images. Psychonomic Bulletin and Review, 14, 989-993.

O'Toole, A. J., Roark, D. A., \& Abdi, H. (2002). Recognizing moving faces: A psychological and neural synthesis. Trends in Cognitive Sciences, 6, 261-266. 
Rajaram, S. (1996). Perceptual effects on remembering: Recollective processes in picture recognition memory. Journal of Experimental Psychology: Learning Memory and Cognition, 22, 365-377.

Ray, C. A., \& Reingold, E. M. (2003). Long-term perceptual specificity effects in recognition memory: The transformed pictures paradigm. Canadian Journal of Experimental Psychology, 57, 131-137.

Reingold, E. M. (2002). On the perceptual specificity of memory respresentations. Memory, 10, 365-380.

Rosenholtz, R., Li, Y. Z., \& Nakano, L. (2007). Measuring visual clutter. Journal of Vision, 7, 1-22.

Rotello, C. M., Macmillan, N. A., \& Van Tassel, G. (2000). Recall-to-reject in recognition: Evidence from ROC curves. Journal of Memory and Language, 43, 67-88.

Szekely, A., D Amico, S., Devescovi, A., Federmeier, K., Herron, D., Iyer, G., et al. (2005). Timed action and object naming. Cortex, 41, 7-26.

Tulving, E. (1983). Elements of episodic memory. Oxford: Oxford University Press. Yonelinas, A. P. (2002). The nature of recollection and familiarity: A review of 30 years of research. Journal of Memory and Language, 46, 441-517. 
Dynamic superiority effect 15

\section{Footnote}

${ }^{1}$ The fact that study-test congruence yielded higher discriminability for each study mode does not imply that study-test congruence yields the best performance across all study and test modes. Indeed, $d_{a}$ in the incongruent moving/multi-static condition was (non-significantly) higher than in the congruent multi-static/multi-static condition in Experiment $1[t(71)=1.41, p=.16]$. 
Dynamic superiority effect 16

\section{Author Notes}

The research in this article was supported by ESRC Grant RES-000-22-2694 (with $\mathrm{KL}$ as $\mathrm{PI})$. 
Figure Captions

Figure 1. Mean discriminability as a function of study and test modes (Experiment 1). Bars are standard errors adjusted for within-subject designs (Masson \& Loftus, 2003).

Figure 2. Mean discriminability as a function of study and test modes (Experiment 2, inclusion condition: respond "old" if test probe is an old scene, regardless of presentation mode). Bars are adjusted standard errors for within-subject designs.

Figure 3. Mean discriminability as a function of study and test modes (Experiment 2, exclusion condition: respond "old" if test probe is an old scene in its original presentation mode). Bars are adjusted standard errors for within-subject designs. 
Figure 1

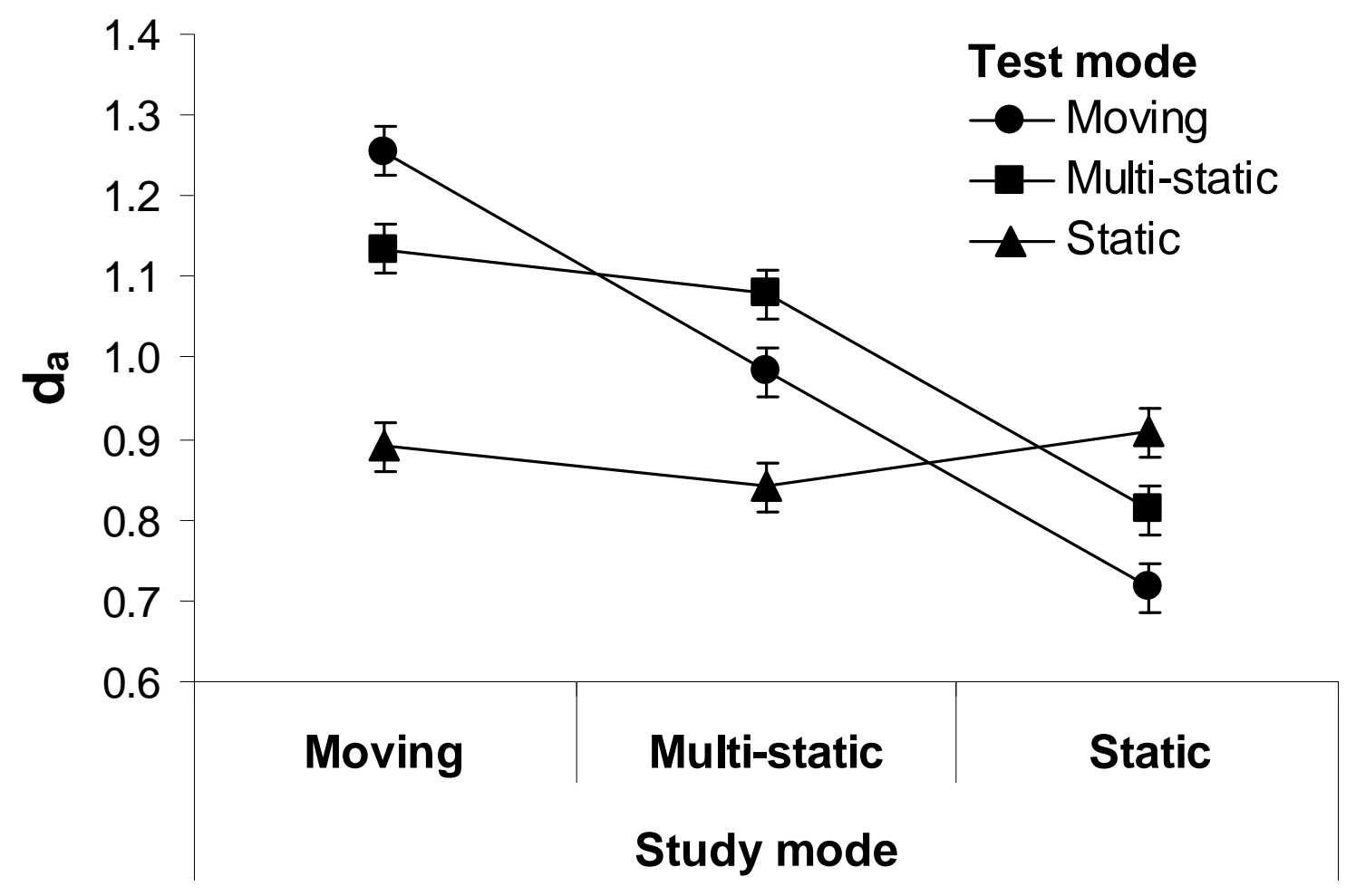


Figure 2

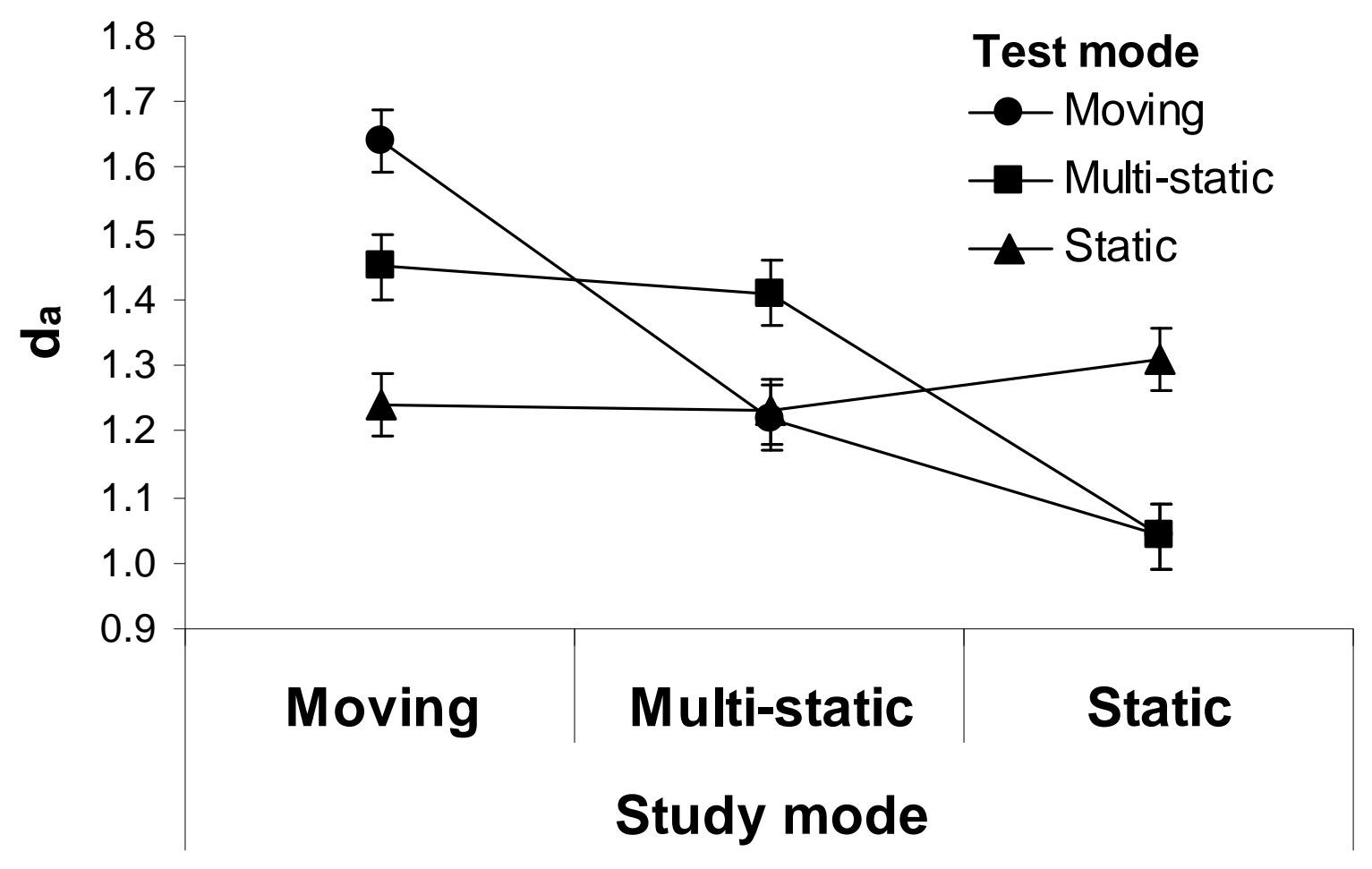


Figure 3

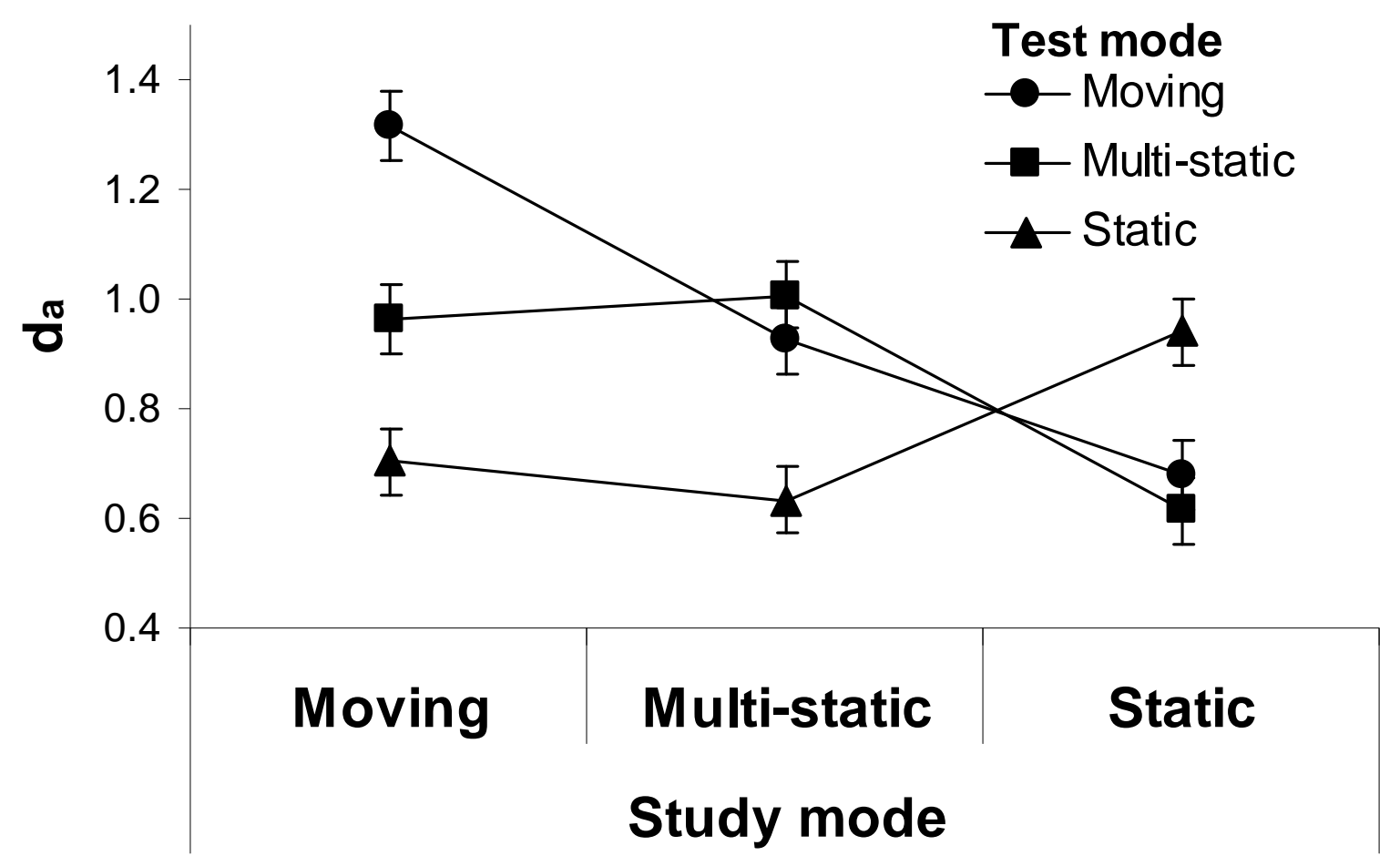

